\title{
Familial olivopontocerebellar atrophy with neonatal onset: a recessively inherited syndrome with systemic and biochemical abnormalities
}

\author{
BRIAN N HARDING,${ }^{*} \dagger$ DAVID B DUNGER, $\dagger$ DAVID B GRANT,$\dagger$ \\ MAGDA ERDOHAZI $\dagger$ \\ From the Institute of Neurology, National Hospitals for Nervous Diseases* and The Institute of Child Health, \\ Hospitals for Sick Children, $†$ London, UK
}

SUMMARY Clinical and pathological findings are reported in two siblings who presented in the neonatal period with failure to thrive, hypotonia, pericardial effusions, limitation of joint movement, retinal dystrophy and loss of visual function. Additional features were biochemical evidence of purine overproduction and liver dysfunction. Post mortem, the neuropathological findings in both children were typical of olivopontocerebellar atrophy. It is suggested that the cases represent a recessively inherited inborn error of metabolism.

Definitive diagnosis of olivopontocerebellar atrophy (OPCA) is morphological, requiring the demonstration of degeneration in the cerebellar cortex and its afferent pathways, in contrast to the dentate nucleus and efferent pathways which are usually spared. Since the original descriptions of sporadic ${ }^{1}$ and dominantly inherited $^{2}$ forms, a large number of somewhat heterogeneous examples of OPCA have been reported. In an attempt to classify these cases on clinical, pathological and genetic grounds, Konigsmark \& Weiner ${ }^{3}$ divided OPCA into five subtypes, all autosomal dominant except type II cases which were recessive or sporadic. Recently, a putative biochemical defect has been identified in some patients with recessive or sporadic OPCA, deficiency of the enzyme glutamate dehydrogenase ${ }^{4}$ which is involved in the metabolism of the excitatory neurotransmitter glutamate. Other neurotransmitter abnormalities have been described in dominant OPCA. ${ }^{6}$

OPCA is rare in childhood, and very few present in the first year of life. To date, all the childhood cases have been part of dominant pedigrees. ${ }^{710}$ We have had the opportunity to examine two siblings presenting a novel and distinctive syndrome with presumed autosomal recessive inheritance, in which

Address for reprint requests: Dr B N Harding, Department of Neuropathology, Hospital for Sick Children, Great Ormond Street, London WCIN 3JH, UK.

Received 14 August 1987.

Accepted 15 September 1987
OPCA is combined with systemic and biochemical abnormalities.

\section{Case reports}

Case 1 was the first child of unrelated Caucasian parents. She was born at term, by normal delivery, weighing $2 \cdot 8 \mathrm{~kg}$. Apgar scores were satisfactory but after 48 hours she was transferred to the special care baby unit with lethargy, hypothermia and feeding problems. Clinical suspicions of septicaemia were not confirmed by screening but she continued to show failure to thrive. There was obvious oedema of the lower limbs and hepatomegaly, and a pericardial effusion was detected by 2D-Echo when she was 5 weeks old. Weight gain remained poor, diarrhoea was an additional problem, and protein losing enteropathy was suspected. She required several plasma transfusions because of oedema. There was also concern about her vision, when roving eye movements were observed. At the age of 6 months, she was transferred to the Hospital for Sick Children, for investigation of her failure to thrive. Her weight was $3.3 \mathrm{~kg}$, length $55 \mathrm{~cm}$, head circumference $38.5 \mathrm{~cm}$; all measurements were well below the 3 rd centile. She was slow to feed, wasted, lethargic, generally hypotonic and developmentally retarded. There were no dysmorphic features. She had occasional roving nystagmoid eye movements, but fixed well and could follow light. Ophthalmoscopic examination showed pale but not hypoplastic discs and pale fundi, diagnosed as retinal dystrophy. The liver edge was palpable $2-3 \mathrm{~cm}$ below the costal margin. Limitation of hip abduction was also noted.

Chest radiographs confirmed the presence of a small pericardial effusion, and abdominal ultrasound demonstrated hepatomegaly and ascites. The EEG was normal. The ERGs had abnormally small amplitude and unusual waveforms, 
while the VERs were also abnormal. CT scan showed prominent occipital and temporal horns and widening of subarachnoid spaces over the occipital region and medial parietal sulci. The vallecula was also prominent. Biochemical investigations revealed low levels of serum albumin and raised transaminases indicative of liver dysfunction. Subsequently she remained lethargic, requiring tube feeding. Plasma proteins dropped, so she needed plasma transfusions. Her condition rapidly deteriorated with increasing oedema and radiological evidence of pneumonia. Ascites was pronounced and she developed increasing tachypnoea. The diarrhoea continued, but jejunal biopsy and ${ }^{51} \mathrm{Cr}$ labelled albumin excretion test were normal. She died aged 7 months.

Case 2 The second child, a male, was also born at term after an uneventful pregnancy and delivery, weighing $2.9 \mathrm{~kg}$. Poor feeding and slow weight gain were noted from birth and on the 11th day he was admitted to hospital with recurrent vomiting and diarrhoea. He was referred to the Hospital for Sick Children at 3 weeks: his weight was $2.6 \mathrm{~kg}$, head circumference $35 \mathrm{~cm}$. There were no dysmorphic features, although his appearance was remarkably similar to that of his sister, with rather widely spaced nipples, long fingers and toes, a depressed nasal bridge and slightly long philtrum. Mild flexion contractures of the knees had developed and hip abduction was limited. Mild hepatomegaly was noted. Neurological examination was normal except that he did not fix or follow. Fundal examination revealed pale fundi and an abnormal greyish appearance to the optic discs.

Biochemical abnormalities were similar to the sister as were EEG, VER and ERG. At 10 weeks of age 2D-Echo demonstrated a moderate pericardial effusion and normal intracardiac anatomy. CT scan demonstrated a large vallecula and fourth ventricle with prominent posterior fossa cisterns. Chromosome analysis was normal. On review at 8 months his weight was $4.32 \mathrm{~kg}$, length $61 \mathrm{~cm}$, and head circumference $41.5 \mathrm{~cm}$; all these measurements were below the 3 rd centile. There was convergent strabismus, no fixing or following, and the optic discs appeared hypoplastic. Tone was increased in all limbs: the hips could not be fully abducted or knees fully extended. Developmental progress was clearly delayed, but slow rather than regressive. Weight gain remained slow and he died aged 23 months from septicaemia.

\section{Biochemical investigations}

Similar abnormalities were noted in both children. Transaminases were raised, indicative of liver dysfunction. Albumin levels were low in Case 1 , but not in Case 2. Both siblings had low levels of thyroxin (3.3 and $3.1 \mathrm{nmol} / \mathrm{l})$, yet TSH and free T4 were normal. Thyroid binding globulin levels were low, as were caeruloplasmin levels. Plasma aminoacids, ammonia and urinary organic acids were normal. Levels of plasma urate were consistently elevated in

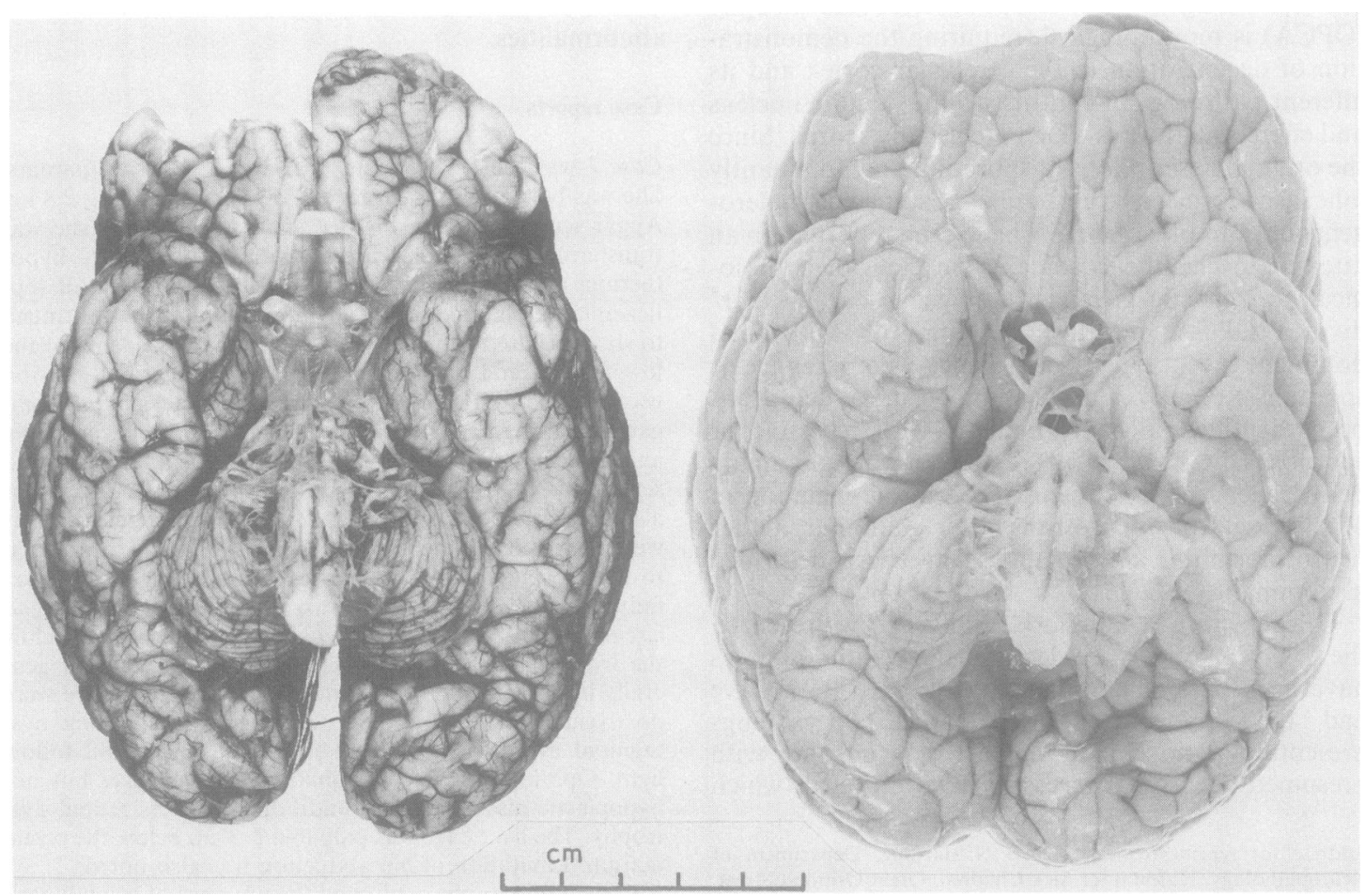

Fig 1 Case 1 (Age at death 7 months) on the left. Case 2 (Age at death 23 months) on the right. Basal view of the fixed brains. Note the particularly severe shrinkage of the cerebellum and the prominence of the folia, as well as the flattening of the ventral pons. (Parts of the right frontal lobes and cerebellum in Case 2 have been removed prior to fixation). 


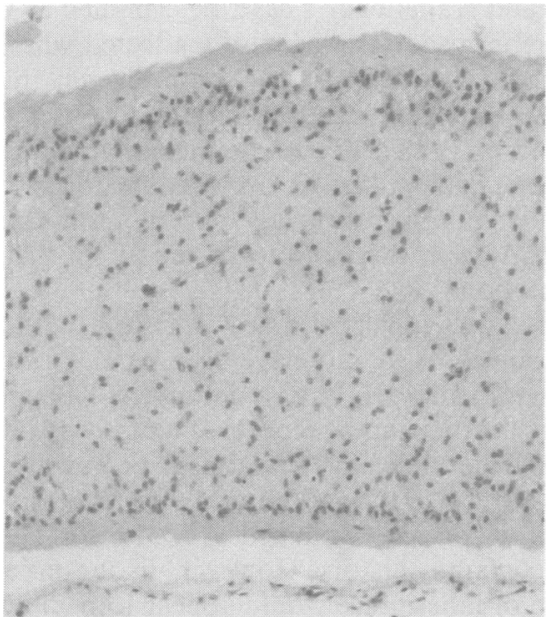

Fig 2 Photomicrograph of a folium from the cerebellar hemisphere. There is complete loss of Purkinje and granule cells and severe gliosis. (Haematoxylin and eosin $\times 110$ ).

both patients $(0.42$ and $0.44 \mathrm{nmol} / \mathrm{l})$.

Further investigation in Case 2 demonstrated a high uric acid/creatinine ratio in the urine $(2 \cdot 8)$ suggestive of purine overproduction. Activity of hypoxanthine-guaninephosphoribosyl transferase (HGPRT) was in the normal range $(110 \mu \mathrm{mol} / \mathrm{ng} / \mathrm{Hb}$ : normal $8-130)$, but levels of PP ribose $P$ synthetase were high, $95 \mathrm{nmol} / \mathrm{mg} / \mathrm{Hb}$ (normal 40-90). Levels of hypoxanthine $(100 \mu \mathrm{mol} / \mathrm{l})$ and xanthine $(31 \mu \mathrm{mol} / \mathrm{l})$ were also elevated supporting the concept of a defect associated with purine overproduction.

Plasma bile salts were analysed in Case 2: levels of chenodeoxycholic acid $(46.63 \mu \mathrm{mol} / 1(\mathrm{~N}=0.22-12.4)$ and cholic acid $(17.44 \mu \mathrm{mol} / 1(\mathrm{~N}=0.06-4.55)$ were raised, compatible with liver dysfunction. Trihydroxycoprostanic acid was not detected. Glutamate metabolism was investigated in two ways. Plasma amino acids were analysed following a standard oral protein load $(\mathrm{lg} / \mathrm{kg})$. Methionine levels $(1086 \mu \mathrm{mol} / \mathrm{l})$ were elevated during the initial protein load but normal in a repeat test. Plasma levels of ketoglutarate were normal during both tests. Also, normal activity of glutamate dehydrogenase was demonstrated histochemically on liver biopsy (Prof B D Lake).

\section{Pathological findings}

At necropsy, both children had evidence of marked hepatic portal fibrosis and cystic dilatation of the distal renal tubules, pulmonary oedema and pericardial effusions. The cardiovascular system was morphologically unremarkable. Neuropathological findings were similar in both cases and will be described together. Both brains were reduced in size and weight, and the hindbrains were relatively more affected. Case 1: total weight $510 \mathrm{~g}$ (normal for age $690 \mathrm{~g}$ ): hindbrain $27 \mathrm{~g}=5 \%$ of total weight (normal 9\%). Case 2: total weight $910 \mathrm{~g}$ (normal $1060 \mathrm{~g}$ ): hindbrain $335 \mathrm{~g}=4 \%$ of total weight (normal $12 \%$ ). The cerebral hemispheres were symmetrical with a normal gyral pattern, and on sectioning were unremarkable. The cerebellum in each case was very small and shrunken with prominent hard folia, and the pons was also small with flattening of the base (fig 1). On sectioning the cerebellar folia were thin and white, and the dentate nuclei difficult to define. The basis pontis was shallow and very pale, while in the medulla the region of the olives was firm and white.

Histologically, the cerebellar cortex in both cases was devastated. Loss of Purkinje and granule cells (fig 2) was almost total except in the nodulus in both brains where a few Purkinje cells with abnormal dendritic expansions remained (fig 3). The residual cerebellar cortex was thin, severely gliotic and virtually devoid of axons. There was no evidence of an external granule layer in Case 1 as would normally be expected at 7 months of age. The medullary white matter was severely depleted of myelin and axons: the dentate amicula appeared worst affected while the hila were better preserved (fig 4). The dentate nuclei themselves showed only mild cell loss and gliosis (fig 5). The superior cerebellar peduncles were intact but the fibres of the middle and inferior peduncles were greatly depleted (fig 4). There was severe neuronal loss and gliosis in the nuclei pontis, virtual disappearance of the transverse pontine fibres (fig 4), and almost total neuronal loss from the olives with heavy gliosis (fig 6). Elsewhere in Case 1 there was only very mild cell loss in the cerebral cortex. Substantia nigra, thalamus and basal ganglia, brainstem tegmentum and upper cervical cord were

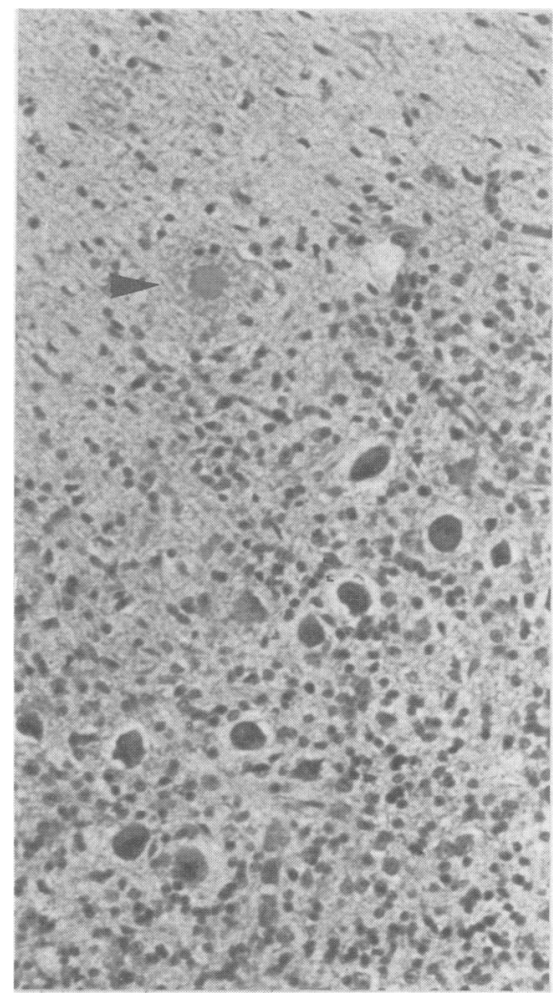

Fig 3 Cerebellar nodulus. A few granule cells and degenerate Purkinje cells remain. Note the abnormal Purkinje dendritic expansion (asteroid body-cactus body). (Arrowhead). (Haematoxylin and eosin $\times 200$ ). 


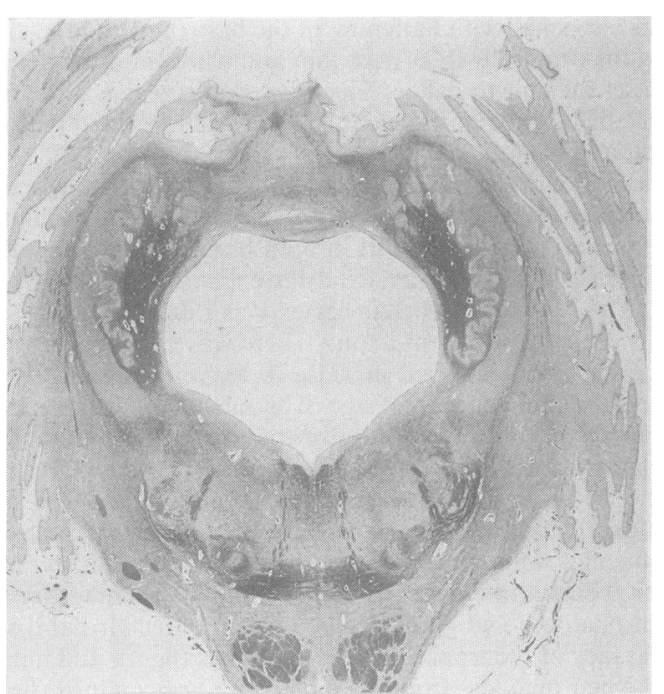

Fig 4 Low power photomicrograph of a horizontal section through the cerebellum and pons stained for myelin. The cerebellar folia are shrunken, the cerebellar peduncles are narrowed, and the ventral pons is shallow. There is severe loss of myelin fibres from the cerebellum and middle cerebellar peduncles, and disappearance of transverse pontine fibres, but the dentate hila are well preserved. (Luxol fast blue, Cresyl violet $\times 21 / 2$ ).

unremarkable. In Case 2 there was generalised moderate neuronal loss and gliosis in the cerebral cortex, and variable, mild to moderate, nerve cell depletion in the basal ganglia and thalamus. Optic nerves and tracts and the lateral geniculate nuclei were unremarkable.

\section{Discussion}

The neuropathological findings in both our cases are typical of OPCA. The major damage is sited in the cerebellar cortex and its afferent fibre pathways and nuclei (inferior olives and nuclei pontis), whereas the cerebellar efferent pathway (dentate nucleus and superior cerebellar peduncle) is spared. Morphological changes are remarkably similar in both siblings, except for more generalised neuronal damage in the cerebral hemisphere of the longer survivor. While this might point to non-specific secondary changes, the same basic defect may underlie both cerebellar and cerebral pathology.

OPCA rarely presents in childhood. Berciano ${ }^{11}$ in a review of 117 reported cases found the average age of onset to be 28 years, and included only two cases ${ }^{12} 13$ presenting in the neonatal period. However, the morphological changes in the first, Norman and Urich's case, are not those of OPCA, being more akin to pontocerebellar hypoplasia. Nor can the second,
Gross and Kaltenback's case, be classified as OPCA: the cerebellar cortex was hardly affected whereas the dentate nuclei and red nuclei were severely involved. Of other reported cases of childhood onset OPCA, none have presented in the neonatal period; the youngest, patient 4 of Colan, ${ }^{7}$ lost head control and became hypotonic at the age of 5 months. Our cases of OPCA seem therefore to be unique in their early presentation.

Most adult inherited cases of OPCA, and all the childhood cases to date have been part of autosomal dominant pedigrees. In a recent review Koeppen and Barron ${ }^{14}$ criticise most reports of recessively inherited OPCA from clinical, pathological or genetic standpoints, although they admit that some recessive cases may be incorrectly assumed sporadic for lack of a complete family history. However, in our patients the pattern of inheritance would appear to be autosomal recessive, although there is an important caveat. There are no other children in the family, and both parents are to the best of our knowledge neurologically normal. But they are relatively young: both are still under 35 years of age. In a few families, with autosomal dominant OPCA and retinal degener-

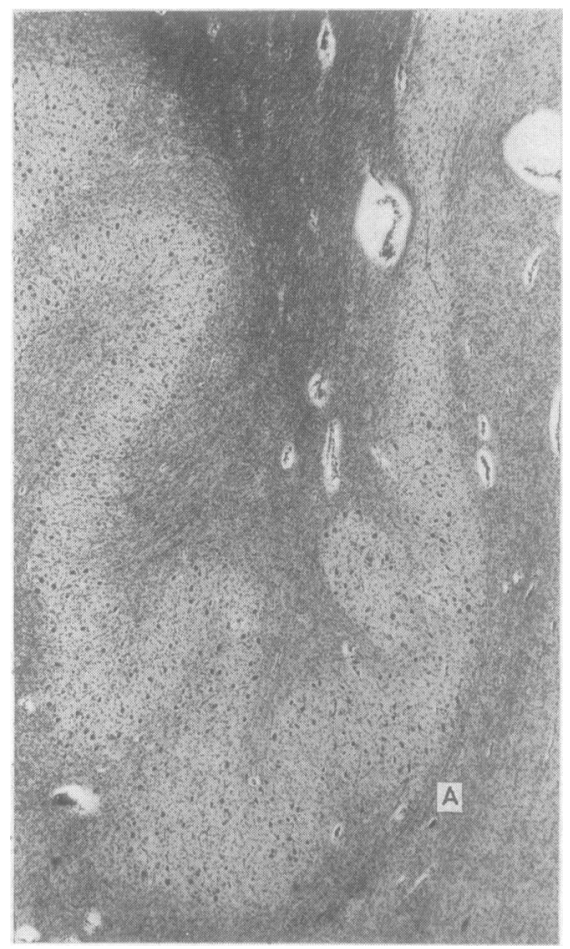

Fig 5 Dentate nucleus. There is good preservation of dentate neurons and of their myelinated fibres in the hilum, but marked loss of fibres from the amiculum (A). (Luxol fast blue, Cresyl violet $\times 30$ ). 


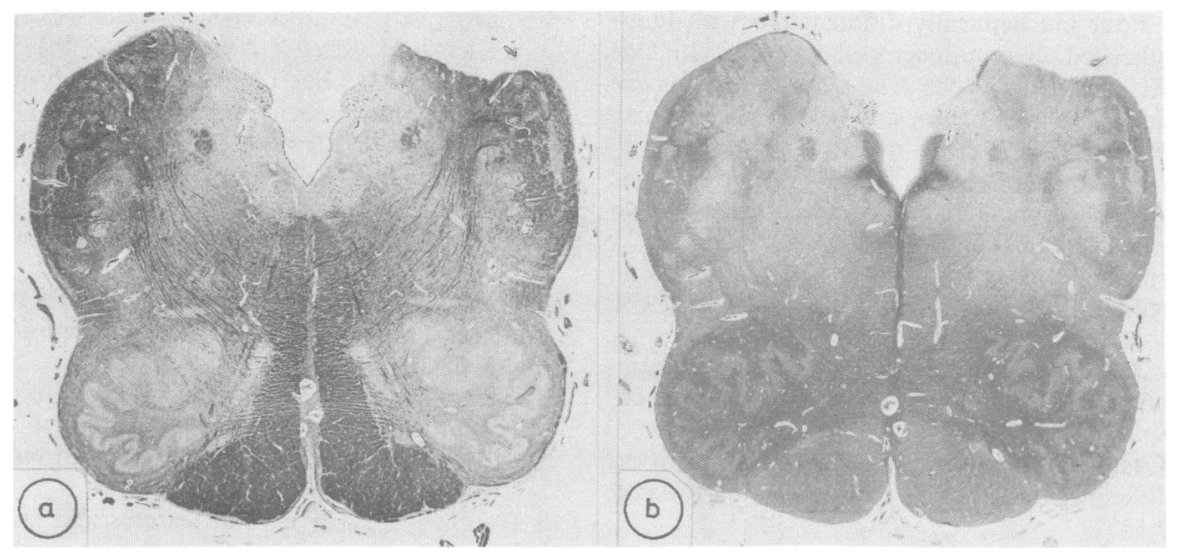

Fig 6 Horizontal section of the medulla stained for myelin (a) and glial fibres (b). Note the myelin loss and gliosis of the olives and olivo-cerebellar fibres. (a) Luxol fast blue, Cresyl violet $\times 5,(b)$, Phosphotungstic acid haematoxylin $\times 5$ ).

ation, there has been an enormous disparity in age of onset. Childhood onset was within the first 2 years, parental onset in the second or third decade. ${ }^{7} 9$ 15 The most extreme example ${ }^{10}$ presented in late infancy, her father and aunt at 41 years and 50 years respectively. These cases ${ }^{7-1015-17}$ seem to form a distinct genetic entity from other dominant cerebellar ataxias, ${ }^{18}$ in particular because of their relatively early onset. But none presented neonatally, or showed the systemic abnormalities which were so evident in our patients.

The clinical features of OPCA are those of progressive cerebellar ataxia, as well as tremor, dysarthria, pyramidal and extrapyramidal signs, ophthalmoplegia and retinal degeneration. In our patients, presenting as neonates, neurological deficit was overshadowed by systemic disease, although limitation of joint movement was detected early in both children, a feature not previously reported in OPCA. Systemic disease, vomiting, diarrhoea, failure to thrive, oedema, pericardial effusions, hepatic fibrosis, has to our knowledge not been recorded before in combination with OPCA. And it clearly points towards an underlying biochemical abnormality.

Some cases of recessive OPCA are reported to have had deficiency of glutamate dehydrogenase. ${ }^{45} \mathrm{We}$ were not able to detect any abnormality of glutamic acid metabolism in our patients, although glutamate levels were elevated in one of two protein load tests. The raised levels of methionine, and decreased levels of thyroid binding globulin, caeruloplasmin and albumin could be explained by liver dysfunction and transaminases were elevated. Plasma urate levels were consistently elevated in both siblings, possibly as a consequence of purine overproduction. Spinocerebellar degeneration has been reported in associ- ation with a variant form of hypoxanthine guanine phosphoribosyl transferase deficiency, ${ }^{19}$ but levels of this enzyme were normal in one of our patients. It therefore seems likely that the primary biochemical abnormality in our patients was not directly related to purine metabolism but led to purine overproduction.

So, in conclusion, the remarkable combination in our sibling cases, of neonatal onset OPCA with generalised systemic disease and biochemical abnormalities, is further evidence that inborn errors of metabolism may underlie CNS system degeneration. Current opinion has swung towards that, far from being a specific disease entity, OPCA is a common pathological end point for several clinically and genetically distinct disorders. Our patients appear to demonstrate a new disorder which falls into this group.

The authors are indebted to Mrs Peggy KelmanMcConnachie for the histological preparations and Miss Kathryn Anson for photography.

\section{References}

1 Dejerine J, Thomas A. L'atrophie olivo-pontocérébellèuse. Nouv Iconogr Salpèt 1900;13:330-70.

2 Menzel P. Beitrag zur Kenntnis der hereditären Ataxien und Kleinhirnatrophie. Arch Psychiatr Nervenkr 1891;22:160-90.

3 Konigsmark BW, Weiner LP. The oliovopontocerebellar atrophies: A review. Medicine 1970;49:227-41.

4 Plaitakis A, Nicklas WJ, Desnick RJ. Glutamate dehydrogenase deficiency in 3 patients with spinocerebellar syndrome. Ann Neurol 1980;7:297-303.

5 Chouroverty S, Khedekar R, Derby B, et al. Pathology of olivopontocerebellar atrophy with glutamate dehydrogenase deficiency. Neurology 1984;34:1451-5. 
6 Perry TL. Four biochemically different types of dominantly inherited olivopontocerebellar atrophy. In: Duvoisin RC, Plaitakis A, eds. The Olivopontocerebellar Atrophies. Advances in Neurology Vol 41. New York: Raven Press, 1984:205-16.

7 Colan RV, Snead OC, Ceballos R. Olivopontocerebellar atrophy in children: a report of seven cases in two families. Ann Neurol 1981;10:355-63.

8 Woodworth JA, Beckett RS, Netsky MG. A composite of hereditary ataxias. Arch Intern Med 1959; 104:594-606.

9 Carpenter S, Schumacher GA. Familial infantile cerebellar atrophy associated with retinal degeneration. Arch Neurol 1986;14:82-94.

10 Amit R, Granit G, Shapira Y. Familial ataxia with extreme difference in age of clinical onset. Neuropediatr 1986;17:165-7.

11 Berciano J. Olivopontocerebellar atrophy: A review of 117 cases. $J$ Neurol Sci 1982;53:253-72.

12 Norman RM, Urich H. Cerebellar hypoplasia associated with systemic degeneration in early life. $J$ Neurol Neurosurg Psychiatry 1958;21:159-66.

13 Gross H, Kaltenbäck E. Über eine Kombinierte progressive pontocerebellare Systematrophie bei einen Kleinkind. Dtsch Z Nervenheilk 1959;179:388-400.

14 Koeppen AH, Barron KD. The Neuropathology of Olivopontocerebellar Atrophy. In: Duvoisin RC, Plaitakis A, eds. The Olivopontocerebellar Atrophies. Advances in Neurology, Vol 41. New York: Raven Press, 1984:13-38.

15 Havener WH. Cerebellar-macular abiotrophy. Arch Ophthal 1951;95:40-43.

16 Forster JB, Ingram TTS. Familial cerebromacular degeneration and ataxia. $J$ Neurol Neurosurg Psychiatry 1962;25:67-68.

17 Weiner LP, Stoll J, Magladery JW. Hereditary olivopontocerebellar atrophy with retinal degeneration. Report of a family through 6 generations. Arch Neurol 1967;16:364-76.

18 Harding AE. The clinical features and classification of the late onset autosomal dominant cerebellar ataxias. Brain 1982;105:1-28.

19 Nyhan WL. Ataxia and disorders of purine metabolism: defects in hypoxanthine guanine phosphoribosyl transferase and clinical ataxia. Adv Neurol 1978;21: 279-87. 